\title{
La lealtad de los estudiantes de la Universidad Nacional de Chimborazo, Riobamba - Ecuador
}

Wilson Saltos AguilaR ${ }^{1}$ Stalin Arguello Erazo ${ }^{2}$ TEONILA GARCÍA ZAPATA ${ }^{3}$

\section{RESUMEN}

El trabajo investigativo hace referencia a los servicios que perciben los estudiantes de la Universidad Nacional de Chimborazo, para conocer las causas y niveles de lealtad, identificando los factores de riesgo y éxito de la calidad de los servicios educativos. El pronóstico de lealtad se basa en los siguientes componentes: Elementos Tangibles, Capacidad de respuesta, Empatía, Fiabilidad, y Seguridad, (Modelo Serqual), dando respuesta a esta constante necesidad de mejorar para lograr la distribución de frecuencias de los niveles de lealtad cognitiva, conativa, afectiva y acción; estudiantes satisfechos que distinguen servicios educativos de calidad.

Palabras clave: Calidad, modelo servqual, lealtad.

LOYALTY OF THE STUDENTS OF THE NATIONAL UNiVERSITY OF CHIMBORAZO, RiobaMba ECUADOR

\section{ABSTRACT}

The research work refers to the services perceived by the students of the National University of Chimborazo, to know the causes and levels of loyalty, identifying the risk factors and success of the quality of educational services. The loyalty forecast is based on the following components: Tangible Elements, Responsiveness, Empathy, Reliability, and Safety, (Servqual Model), responding to this constant need to improve to achieve the frequency distribution of levels of cognitive loyalty, Conative, affective and action; Students who distinguish quality educational services.

Keywords: Quality, servqual model, loyalty.

\section{INTRODUCCIÓN}

La lealtad se entendió inicialmente como compras repetidas (Oliver, 1999), sin embargo, la idea de las compras repetidas no captura todo el dominio de la construcción (Newman \& Werbal, 1973), (Oliver, 1999) define la lealtad de los consumidores como un profundo compromiso para recomprar o repatronise una marca de producto/servicio preferido sistemáticamente en el futuro, a pesar de las influencias situacionales y/o esfuerzos de marketing que puede actuar como causa para cambiar el comportamiento.

(Sirdeshmukh \& Sabol, 2002), afirman que "la lealtad del consumidor se indica con la intención de realizar un conjunto diverso de conductas que indican una motivación para mantener una relación con la empresa focal, incluyendo la asignación de una mayor proporción de la categoría de cartera para el servicio específico proveedor, la participación en la palabra positiva de la boca, y repetir la compra" (p.32).

(Milikan, 2007), utiliza una buena metáfora para describir el escenario bajo la lealtad del estudiante-cliente con respecto a la educación: las epidemias. Esta es una epidemia que afecta a la supervivencia de muchas instituciones de educación en todos los niveles. La educación superior ha estado viviendo esta realidad desde hace mucho tiempo, pero, desde las últimas décadas del siglo 20 , que ha recaudado más atención, como elementos tales como la globalización y la competencia han contribuido a su importancia. Según (Schwartzman, 2003), la deserción se ha convertido en uno de los temas más importantes con respecto a la supervivencia de la Institución de Educación Superior.

Ahora se trata como una tendencia en este sector, junto con la delincuencia, las aberturas vacías y los estudiantes de las clases menos bajas. Por lo tanto, existe esta necesidad implacable para retener a los estudiantes que ya están inscritos. Según lo definido por (Berger, \& Lyon, 2005), la retención en el contexto de la educación superior, es la "capacidad de una escuela o universidad para graduarse con éxito a los estudiantes que se

Master en Finanzas. Docente Principal de la Universidad Nacional de Chimborazo, Ecuador; Doctorado en Gestión de Empresas de la Facultad de Ingeniería Industrial, UNMSM, Lima Perú. E-mail: wsaltos@unach.edu.ec

2 Doctor en Gestión Empresarial. Docente Tiempo Completo de la Escuela Superior Politécnica de Chimborazo, Ecuador.

E-mail: stalin.arguello@espoch.edu.ec

3 Doctora en Ingeniería Industrial. Profesor Principal de la Facultad de Ingeniería Industrial de la UNMSM.

E-mail: tgarciaz@unmsm.edu.pe 
han matriculado en la institución en el primer lugar" (p.92). (Kotler, \& Fox, 1994), no puede hacer suficiente hincapié al decir que retener a los estudiantes matriculados es tan importante como atraer e inscribir a ellos.

En opinión de (Herzog, 2005), el enfoque de la retención de estudiantes en el cliente es "un reto, tanto teóricamente y en términos de funcionamiento institucional" (p.905). En este contexto, (Mclaughlin, \& Brozovsky, \& Mclaughlin, 1998), defienden la idea de que la retención de los estudiantes es un objetivo estratégico, seguido de un profundo cambio en la cultura organizacional de la Institución de Educación Superior, el tratamiento de los estudiantes-clientes en realidad como partes interesadas.

(Mortenson, 2005), llama a las decisiones de retención de los estudiantes en la persistencia de la institución. Estos términos se presentan un problema de tiempo con respecto a la retención de estudiantes en el cliente.

Si bien la planificación de medición persistencia y, por lo tanto, la retención, el investigador debe responder a las siguientes preguntas: ¿Cómo son los estudiantes yendo la trayectoria académica? ¿Quién lo está haciendo bien y quién no lo es? Los estudiantes persisten a lo largo de los años? ¿Dónde hay que mejorar su persistencia? La información utilizada para responder a estas preguntas viene de los datos de matriculación de los estudiantes y su rendimiento académico en puntos definidos en el tiempo.

Esta información, obviamente, se debe cruzar con los datos demográficos y geográficos con respecto a los estudiantes encuestados (Wetzet, \& Peterson., 1999).

(Mortenson, 2005), la calidad educativa es un concepto bastante impreciso y controvertido en la investigación y la política de la discusión. Para diferentes personas, la definición puede variar por lo que los indicadores que se utilizan para describir la calidad de la educación pueden ser diferentes (Fuller, 1986), algunos pueden hacer hincapié en la calidad de los insumos para los sistemas de educación, mientras que otros hacen hincapié en la calidad del proceso y los resultados.

Si se refiere a insumos, procesos, resultados, o todos ellos, la definición de la calidad de la educación a menudo se puede asociar a la aptitud para el uso, la satisfacción de las necesidades de los grupos estratégicos (por ejemplo, los responsables políticos, padres, comité de gestión de la escuela, profesores, estudiantes, etc.) o de conformidad con los requisitos y expectativas circunscripciones estratégicas (Cheng, 1997).
El préstamo de las ideas de enfoque total del sistema de gestión y calidad, (Cheng, 1995) define la calidad de la educación de la siguiente manera: $\mathrm{Ca}-$ lidad de la educación es el carácter del conjunto de elementos de la entrada, proceso y salida del sistema educativo que ofrece servicios que satisfacen por completo tanto grupos estratégicos internos y externos mediante el cumplimiento de sus expectativas explícitas e implícitas.

La revisión de la literatura reveló dos estudios con el uso de SERVQUAL en la educación superior. (Boulding \& Kalra \& Staeling \& Zeithaml, 1992), utilizaron SERVQUAL en un estudio para probar un modelo de proceso de la calidad del servicio. El equipo de investigación utilizó un SERVQUAL modificada de 36 ítems para capturar las expectativas y las percepciones asociadas a un servicio educativo.

Importante a la educación, los autores postulan que las mayores percepciones de la calidad general del servicio de una empresa de los clientes, es más probable que estos clientes estarían a participar en comportamientos beneficiosos para la salud estratégica de la empresa o una universidad.

Al contrastar lo que el cliente cree una firma proveerá con lo que se debe proporcionar, Boulding et al. Encontró que: el aumento de las expectativas del cliente de lo que una empresa prestará durante futuros encuentros de servicio en realidad conduce a una mayor percepción de la calidad después de que el cliente está expuesto al servicio real, todo lo demás es igual resultados apoyan firmemente nuestra premisa de que las expectativas de lo que una empresa de los clientes debe entregar durante un encuentro de servicio a reducir sus percepciones últimos del servicio efectivo entregado.

(Parasuraman \& Zeithaml \& Berry, 1988) definen la calidad del servicio como "un juicio global o actitud en relación con la excelencia en general o la superioridad del servicio" (p.22). y conceptualizan la evaluación de un cliente de la calidad general del servicio mediante la aplicación (1980) modelo de desconfirmación de Oliver, la brecha entre las expectativas y la percepción (modelo de la brecha) de los niveles de rendimiento del servicio.

Además, proponen que el rendimiento global de la calidad de servicio puede ser determinado por una escala de medida llamada "SERVQUAL", que utiliza cinco dimensiones genéricas: Tangibles, Fiabilidad, Capacidad de respuesta, Seguridad, Empatía.

El propósito de este trabajo investigativo es establecer los factores de éxito y riesgo de la Lealtad de los estudiantes de las diferentes carreras que 
oferta la Universidad Nacional de Chimborazo de la ciudad de Riobamba Ecuador.

\section{METODOLOGÍA}

(Hernandez \& Fernandez \& Baptista, 2010), describe en su libro los siguientes tipos de investigación descriptiva, exploratoria y correlacional.

La investigación fue descriptiva porque buscó propiedades, características y rasgos importantes de la situación o fenómeno en estudio, así como las necesidades de los estudiantes de la Universidad Nacional de Chimborazo Riobamba-Ecuador, permitió diagnosticar e identificar las características de las variables de estudio, así también describir las tendencias de la población en estudio.

Fue exploratoria ya que manifiestan (Hernandez \& Fernandez \& Baptista, 2010) que estos estudios se los realizan cuando el objeto consiste en examinar un tema o problema poco estudiado, por lo que en la Universidad Nacional de Chimborazo no se han realizado estudios de esta naturaleza, permitiendo así identificar las necesidades de los estudiantes en la calidad de sus servicios percibidos que no son atendidos, para identificar sus problemas y lograr corregirlos para mejorarlos y lograr lealtad y la sugerencia para futuros clientes (estudiantes).

La valoración de los atributos de la marca y los servivios debe ser preferida a las ofertas competitivas. Es una valoración puramente cognitiva. Como se ha identificado para señalar la lealtad, a las características y atributos de los servicios educativos.

la lealtad afectiva se ha determinada por la insatisfacción cognitivamente inducida (comparación entre expectativas u resultados).

Los determinantes de la lealtad conativa serian la lealtad afectiva y la insatisfacción afectivamente generada por la calidad de servicios educativos recibidos por los estudientes de la Universidad Nacional de Chimborazo.

La acción, supone la lealtad a una inercia de acción junto a una superación de los obstáculos de los servicios educativos ofrecidos por la Institución de Educación Superior (UNACH). Fue correlacional porque asocia y desea conocer la relación entre las variables en análisis de la calidad de los servicios educativos ofertados por la Universidad Nacional de Chimborazo a los estudiantes, para qué de esta manera lograr lealtad y mantener la eficiencia, eficacia y la efectividad en los procesos de oferta de los servicios.
Dónde:

X: Calidad de los Servicios.

Dimensiones de la calidad de los servicios según el modelo SERVQUAL:

X1: Elementos Tangibles, X2: Fiabilidad, X3: Capacidad de Respuesta, X4: Empatía, X5: Seguridad.

Y: Lealtad de los estudiantes de la UNACH.

Y1: Cognitiva, Y2: Conativa, Y3: Afectiva, Y4: Acción.

El presente estudio tiene como unidad de análisis a los estudiantes de la Universidad Nacional del Chimborazo de tal forma que reúnan las condiciones para evaluar los servicios educativos y luego realizar un diagnóstico de la calidad de los servicios que brinda a los estudiantes.

La población está conformada por todas las carreras profesionales de la Universidad Nacional del Chimborazo y el instituto de posgrado, cuya totalidad es de 8377 estudiantes.

La técnica de muestreo fue aleatoria, el procedimiento para elegir a los participantes de la muestra de la siguiente manera: se tomó el código estudiantil de matrícula y de acuerdo a los números seleccionados con el SPSS aplicando la técnica aleatoria; se procederá a tomar el test de lealtad a dichos estudiantes.

Para el tamaño de muestra se utilizó la fórmula de:

$$
n=\frac{Z_{\alpha}^{2} \cdot N \cdot p \cdot q}{i^{2}(N-1)+Z_{\alpha}^{2} \cdot p \cdot q}
$$

Dónde:

$\mathrm{n}=$ Tamaño de la muestra

$\mathrm{N}=$ Tamaño de la población

$Z_{\alpha}=1.96$ al cuadrado (si la seguridad es del 95\%)

$p=$ proporción esperada (en este caso 0.5 )

$q=1-p($ en este caso $1-0,5=0.5)$

$i=\operatorname{error}(5 \%)$

\section{Semestral}

$n=\frac{(1.96)^{2} *(5290) *(0.5) *(0.5)}{(0.0025) *(5290-1)+(1962) *(0.5) *(0.5)} \quad n=335$ 
Anual

$n=\frac{(1.96)^{2} *(2225) *(0.5) *(0.5)}{(0.0025) *(2225-1)+(1962) *(0.5) *(0.5)} \quad n=259$

UFAP

$n=\frac{(1.96)^{2} *(381) *(0.5) *(0.5)}{(0.0025) *(381-1)+(1962) *(0.5) *(0.5)} \quad n=77$

Posgrado

$n=\frac{(1.96)^{2} *(481) *(0.5) *(0.5)}{(0.0025) *(841-1)+(1962) *(0.5) *(0.5)} \quad n=148$

Muestra total 819 estudiantes.

Tabla 1. Análisis de factores de la dimensión de elementos tangibles en la lealtad de los estudiantes de la (UNACH).

\begin{tabular}{|c|c|c|c|c|c|c|}
\hline \multicolumn{7}{|c|}{ Capa: Calidad del Servicio } \\
\hline \multirow{3}{*}{$\begin{array}{l}\text { Factores de la Calidad - } \\
\text { Proceso Deductivo }\end{array}$} & \multicolumn{2}{|c|}{ Est. Inicial } & \multicolumn{2}{|c|}{ Est. Final } & \multirow[b]{2}{*}{ Reg. } & \multirow[b]{2}{*}{ Exc. } \\
\hline & Reg. & Exc. & Reg. & Exc. & & \\
\hline & \multicolumn{4}{|c|}{ Calidad de Servicio } & \multicolumn{2}{|c|}{ Efecto } \\
\hline ELEMENTOS TANGIBLES & 63.00 & 26.50 & 51.80 & 36.20 & -11.20 & 9.70 \\
\hline Limpieza de Oficina & 63.00 & 26.50 & 63.00 & 26.90 & 0.00 & 0.40 \\
\hline Decoración & 63.00 & 26.50 & 63.10 & 26.90 & 0.10 & 0.40 \\
\hline Limpieza SSHH & 63.00 & 26.50 & 58.70 & 29.90 & -4.30 & 3.40 \\
\hline Comodidad de Aula & 63.00 & 26.50 & 62.00 & 27.60 & -1.00 & 1.10 \\
\hline Comodidad de Oficinas & 63.00 & 26.50 & 61.30 & 28.10 & -1.70 & 1.60 \\
\hline Presentación del Personal & 63.00 & 26.50 & 62.80 & 27.10 & -0.20 & 0.60 \\
\hline Presentación del Docente & 63.00 & 26.50 & 63.90 & 26.20 & 0.90 & 0.30 \\
\hline
\end{tabular}

Fuente. Elaboración propia.

Se observa que, al evidenciar o instanciar en el estado de excelencia los factores de limpieza de los servicios higiénicos, comodidad del aula y comodidad de las oficinas se logra un efecto en la calidad de los servicios de 3.40, 1.10 y 1.60 respectivamente. De aquí se deduce que el factor que tiene mayor efecto en la calidad de los servicios es la limpieza de los servicios higiénicos seguidos de la comodidad de las oficinas y luego de la comodidad de las aulas.

También se observa que, al evidenciar o instanciar en el estado de excelencia los factores de limpieza de los servicios higiénicos, comodidad del aula y comodidad de las oficinas el efecto en la lealtad de los estudiantes es de $0.60 ; 0.30$ y 0.30 respectivamente. De aquí se deduce que el factor que tiene

\section{RESULTADOS}

Para la validación del instrumento se utilizó lo siguiente:

Análisis factorial exploratorio: Es una técnica estadística para analizar las interrelaciones o interdependencia entre datos observables, se fundamenta en el análisis de la matriz de correlaciones, por tanto los ítems deben ser cuantitativos, pero la técnica soporta ítems medidos bajo escala ordinal.

En la Tabla 1 se identifica la capa de la calidad de los servicios ofertados por la Universidad Nacional de Chimborazo, dando respuesta a la capa de lealtad por parte de los estudiantes en las diferentes dimensiones de los elementos tangibles. 
Tabla 2. Análisis del efecto de los factores de la capacidad de respuesta en la lealtad de los estudiantes.

\begin{tabular}{|c|c|c|c|c|c|c|}
\hline \multicolumn{7}{|c|}{ Capa: Calidad del Servicio } \\
\hline \multirow{3}{*}{$\begin{array}{l}\text { Factores de la Calidad - } \\
\text { Proceso Deductivo }\end{array}$} & \multicolumn{2}{|c|}{ Est. Inicial } & \multicolumn{2}{|c|}{ Est. Final } & & \\
\hline & Reg. & Exc. & Reg. & Exc. & Reg & Exc. \\
\hline & \multicolumn{4}{|c|}{ Calidad del Servicio } & \multicolumn{2}{|c|}{ Efecto } \\
\hline CAPACIDAD DE RESPUESTA & 63.00 & 26.50 & 54.90 & 33.20 & -8.10 & 6.70 \\
\hline Rapidez de Atención & 63.00 & 26.50 & 60.20 & 29.10 & -2.80 & 2.60 \\
\hline Entrega Puntual de Notas & 63.00 & 26.50 & 61.60 & 28.10 & -1.40 & 1.60 \\
\hline Ayuda con Prontitud & 63.00 & 26.50 & 61.20 & 28.60 & -1.80 & 2.10 \\
\hline Atención de Quejas & 63.00 & 26.50 & 60.40 & 29.00 & -2.60 & 2.50 \\
\hline
\end{tabular}

\begin{tabular}{lccccc}
\hline \multicolumn{5}{c}{ Capa: Lealtad del Estudiante } \\
\hline \multicolumn{2}{c}{ Est. Inicial } & \multicolumn{2}{c}{ Est. Final } \\
\hline Reg. & Exc. & Reg. & Exc. & Reg. Exc. \\
\hline \multicolumn{3}{c}{ Lealtad del Estudiante } & \multicolumn{3}{c}{ Efecto } \\
\hline 44.40 & 49.40 & 42.70 & 50.60 & -1.70 & 1.20 \\
\hline 44.40 & 49.40 & 43.80 & 49.90 & -0.60 & 0.50 \\
44.40 & 49.40 & 44.10 & 49.80 & -0.30 & 0.40 \\
44.40 & 49.40 & 44.00 & 49.90 & -0.40 & 0.50 \\
44.40 & 49.40 & 43.80 & 49.90 & -0.60 & 0.50 \\
\hline
\end{tabular}

Fuente. Elaboración propia.

Se observa que, al evidenciar o instanciar en el estado de excelencia los factores de rapidez de atención y atención de quejas se logra un efecto en la calidad de los servicios de 2.60 y 2.50 respectivamente. De aquí se deduce que el factor que tiene mayor efecto en la calidad de los servicios es la rapidez de atención seguidos por la atención de quejas.

También se observa que, al evidenciar o instanciar en el estado de excelencia los factores de rapidez de atención y atención de quejas el efecto en la lealtad de los estudiantes es de 0.50 y 0.50 respectivamente. De aquí se deduce que ambos factores tienen el mismo nivel de efecto en la lealtad.
Además, el efecto en general en la calidad de los servicios es de 6.70 y en la lealtad es de 1.20; es decir si se brinda un servicio de excelencia en la rapidez de atención y atención de quejas la calidad de los servicios en general incrementa en una probabilidad de 6.70 y la lealtad de los alumnos en 1.20 .

En la Tabla 3 se identifica la capa de la calidad de los servicios ofertados por la Universidad Nacional de Chimborazo, dando respuesta a la capa de lealtad por parte de los estudiantes en las diferentes dimensiones de Empatía.

Tabla 3. Análisis del efecto de los factores de la empatía en la lealtad de los estudiantes.

\begin{tabular}{|c|c|c|c|c|c|c|}
\hline \multicolumn{7}{|c|}{ Capa: Calidad del Servicio } \\
\hline \multirow{3}{*}{$\begin{array}{l}\text { Factores de la Calidad } \\
\text { - Proceso Deductivo }\end{array}$} & \multicolumn{2}{|c|}{ Est. Inicial } & \multicolumn{2}{|c|}{ Est. Final } & \multirow{2}{*}{\multicolumn{2}{|c|}{ Reg. Exc. }} \\
\hline & Reg. & Exc. & Reg. & Exc. & & \\
\hline & \multicolumn{4}{|c|}{ Calidad de Servicio } & \multicolumn{2}{|c|}{ Efecto } \\
\hline EMPATIA & 63.00 & 26.50 & 58.20 & 30.90 & -4.80 & 4.40 \\
\hline Amabilidad y Cortesía & 63.00 & 26.50 & 61.40 & 28.20 & -1.60 & 1.70 \\
\hline Horarios Adecuados & 63.00 & 26.50 & 61.40 & 28.20 & -1.60 & 1.70 \\
\hline Escuchar al Alumno & 63.00 & 26.50 & 61.10 & 28.40 & -1.90 & 1.90 \\
\hline Comprensión & 63.00 & 26.50 & 61.60 & 28.10 & -1.40 & 1.60 \\
\hline
\end{tabular}

\begin{tabular}{cccccc}
\hline \multicolumn{5}{c}{ Capa: Lealtad del Estudiante } \\
\hline \multicolumn{2}{c}{ Est. Inicial } & \multicolumn{2}{c}{ Est. Final } \\
\hline Reg. & Exc. & Reg. & Exc. & \multicolumn{2}{c}{ Reg. Exc. } \\
\hline \multicolumn{3}{c}{ Lealtad del Estudiante } & \multicolumn{2}{c}{ Efecto } \\
\hline 44.40 & 49.40 & 43.40 & 50.30 & -1.00 & 0.90 \\
\hline 44.40 & 49.40 & 44.00 & 49.80 & -0.40 & 0.40 \\
44.40 & 49.40 & 44.10 & 49.80 & -0.30 & 0.40 \\
44.40 & 49.40 & 44.00 & 49.80 & -0.40 & 0.40 \\
44.40 & 49.40 & 44.10 & 49.80 & -0.30 & 0.40 \\
\hline
\end{tabular}

Fuente. Elaboración propia.

Se observa que, al evidenciar o instanciar en el estado de excelencia los factores de Amabilidad y Cortesía, y Escuchar al alumno se logra un efecto en la calidad de los servicios de 1.7 y 1.9 respectivamente.
De aquí se deduce que el factor que tiene mayor efecto en la calidad de los servicios es la Escuchar al alumno seguidos por la Amabilidad y Cortesía.

También se observa que, al evidenciar o instanciar en el estado de excelencia los factores de Amabili- 
dad y Cortesía, y Escuchar al alumno el efecto en la lealtad de los estudiantes es de 0.40 y 0.40 respectivamente. De aquí se deduce que ambos factores tienen el mismo nivel de efecto en la lealtad.

Además, el efecto en general de la empatía en la calidad de los servicios es de 4.4 y en la lealtad es de 0.9; es decir, si se brinda un servicio de excelencia en los factores de Amabilidad y Cortesía, y
Escuchar al alumno, la calidad de los servicios en general incrementa en una probabilidad de 4.4 y la lealtad de los alumnos en 0.9.

En la Tabla 4 se identifica la capa de la calidad de los servicios ofertados por la Universidad Nacional de Chimborazo, dando respuesta a la capa de lealtad por parte de los estudiantes en las diferentes dimensiones de Fiabilidad.

Tabla 4. Análisis del efecto de los factores de la fiabilidad en la lealtad de los estudiantes.

\begin{tabular}{lccccccc}
\hline \multicolumn{7}{c}{ Capa: Calidad del Servicio } \\
\hline \multicolumn{7}{c}{ Est. Inicial } & Est. Final \\
\hline $\begin{array}{l}\text { Factores de la Calidad }- \\
\text { Proceso Deductivo }\end{array}$ & Reg. & Exc. & Reg. & Exc. & Reg. Exc. \\
\cline { 2 - 7 } & 63.00 & 26.50 & 58.60 & 30.80 & -4.40 & 4.30 \\
\hline FIABILIDAD & 63.00 & 26.50 & 60.00 & 29.50 & -3.00 & 3.00 \\
\hline Desempeño del Personal & 63.00 & 26.50 & 60.90 & 28.70 & -2.10 & 2.20 \\
Uso de la tecnología & 63.00 & 26.50 & 58.60 & 30.80 & -4.40 & 4.30 \\
Sabe dónde quejarse & & & & & Efecto \\
\hline
\end{tabular}

\begin{tabular}{cccccc}
\hline \multicolumn{5}{c}{ Capa: Lealtad del Estudiante } \\
\hline \multicolumn{2}{c}{ Est. Inicial } & \multicolumn{2}{c}{ Est. Final } \\
\hline Reg. & Exc. & Reg. & Exc. & \multicolumn{2}{c}{ Reg. Exc. } \\
\hline \multicolumn{3}{c}{ Lealtad del Estudiante } & \multicolumn{2}{c}{ Efecto } \\
\hline 44.40 & 49.40 & 43.40 & 50.30 & -1.00 & 0.90 \\
\hline 44.40 & 49.40 & 43.70 & 50.00 & -0.70 & 0.60 \\
44.40 & 49.40 & 43.90 & 49.90 & -0.50 & 0.50 \\
44.40 & 49.40 & 43.90 & 50.30 & -1.00 & 0.90 \\
\hline
\end{tabular}

Fuente. Elaboración propia.

Se observa que, al evidenciar o instanciar en el estado de excelencia el factor de: sabe dónde quejarse, se logra un efecto en la calidad de los servicios de 4.3. De aquí se deduce que el factor que tiene mayor efecto en la calidad de los servicios es que el estudiante sabe dónde quejarse.

Al evidenciar o instanciar en el estado de excelencia el factor de: sabe dónde quejarse, el efecto en la lealtad de los estudiantes es de 0.90.

Además, el efecto en general de la fiabilidad en la calidad de los servicios es de 4.30 y en la lealtad es de 0.9; es decir, si se brinda un servicio de excelencia en los factor en donde el alumno conoce donde quejarse, la calidad de los servicios en general incrementa en una probabilidad de 4.30 y la lealtad de los alumnos en 0.9.

En la Tabla 5 se identifica la capa de la calidad de los servicios ofertados por la Universidad Nacional de Chimborazo, dando respuesta a la capa de lealtad por parte de los estudiantes en las diferentes dimensiones a la seguridad.

Tabla 5. Análisis del efecto de los factores de seguridad en la lealtad de los estudiantes.

\begin{tabular}{|c|c|c|c|c|c|c|}
\hline \multicolumn{7}{|c|}{ Capa: Calidad del Servicio } \\
\hline \multirow{3}{*}{$\begin{array}{l}\text { Factores de la Calidad - } \\
\text { Proceso Deductivo }\end{array}$} & \multicolumn{2}{|c|}{ Est. Inicial } & \multicolumn{2}{|c|}{ Est. Final } & \multirow{2}{*}{\multicolumn{2}{|c|}{ Reg. Exc. }} \\
\hline & Reg. & Exc. & Reg. & Exc. & & \\
\hline & \multicolumn{4}{|c|}{ Calidad de Servicio } & \multicolumn{2}{|c|}{ Efecto } \\
\hline SEGURIDAD & 63.00 & 26.50 & 62.30 & 27.40 & -0.70 & 0.90 \\
\hline Explicación Clara & 63.00 & 26.50 & 63.00 & 26.90 & 0.00 & 0.40 \\
\hline Exactitud de respuestas & 63.00 & 26.50 & 62.30 & 27.30 & 0.70 & 0.80 \\
\hline Conformidad con el servicio & 63.00 & 26.50 & 61.40 & 28.00 & -1.60 & 1.50 \\
\hline Información útil & 63.00 & 26.50 & 62.50 & 27.30 & -0.50 & 0.80 \\
\hline Seguridad en el campus & 63.00 & 26.50 & 62.70 & 27.00 & -0.30 & 0.50 \\
\hline Experiencia del Docente & 63.00 & 26.50 & 63.30 & 26.60 & 0.30 & 0.10 \\
\hline Orientación & 63.00 & 26.50 & 69.20 & 26.90 & 6.20 & 0.40 \\
\hline Desempeño Docente & 63.00 & 26.50 & 63.00 & 26.70 & 0.00 & 0.20 \\
\hline
\end{tabular}

\begin{tabular}{cccccc}
\hline \multicolumn{6}{c}{ Capa: Lealtad del Estudiante } \\
\hline \multicolumn{2}{c}{ Est. Inicial } & \multicolumn{2}{c}{ Est. Final } \\
\hline Reg. & Exc. & Reg. & Exc. & \multicolumn{2}{c}{ Reg. Exc. } \\
\hline \multicolumn{3}{c}{ Lealtad del Estudiante } & \multicolumn{2}{c}{ Efecto } \\
\hline 44.40 & 49.40 & 44.30 & 49.60 & -0.10 & 0.20 \\
\hline 44.40 & 49.40 & 44.40 & 49.60 & 0.00 & 0.20 \\
44.40 & 49.40 & 44.30 & 49.50 & -0.10 & 0.10 \\
44.40 & 49.40 & 44.10 & 49.60 & -0.30 & $\mathbf{0 . 2 0}$ \\
44.40 & 49.40 & 44.30 & 49.60 & -0.10 & $\mathbf{0 . 2 0}$ \\
44.40 & 49.40 & 44.40 & 49.50 & 0.00 & $\mathbf{0 . 1 0}$ \\
44.40 & 49.40 & 44.50 & 49.50 & 0.10 & 0.10 \\
44.40 & 49.40 & 44.40 & 49.60 & 0.00 & $\mathbf{0 . 2 0}$ \\
44.40 & 49.40 & 37.80 & 56.10 & -6.60 & $\mathbf{6 . 7 0}$ \\
\hline
\end{tabular}

Fuente. Elaboración propia. 
Se observa que, al evidenciar o instanciar en el estado de excelencia el factor de: conformidad con el servicio, se logra un efecto en la calidad de los servicios de 1.5. De aquí se deduce que el factor que tiene mayor efecto en la calidad de los servicios es que cuando el estudiante está conforme con los servicios recibidos.

También se observa que, al evidenciar o instanciar en el estado de excelencia el factor de: información útil, el efecto en la lealtad de los estudiantes es de 0.20 .

Al evidenciar o instanciar en el estado de excelencia el factor de: seguridad en el campus, el efecto en la lealtad de los estudiantes es de 0.50 .

También al evidenciar o instanciar en el estado de excelencia el factor de: orientación, el efecto en la lealtad de los estudiantes es de 0.20.

Al evidenciar o instanciar en el estado de excelencia el factor de: desempeño docente, el efecto en la lealtad de los estudiantes es de 6.70. Que es uno de los factores de mayor efecto.

\section{CONCLUSIONES Y RECOMENDACIONES}

Con respecto a la dimensión de los elementos tangibles del servicios se concluye que, al evidenciar o instanciar en el estado de excelencia los factores de limpieza de los servicios higiénicos, comodidad del aula y comodidad de las oficinas el efecto en la lealtad de los estudiantes es de $0.60,0.30$ y 0.30 respectivamente. De aquí se concluye que los factores de los elementos tangibles tiene un efecto positivo en la lealtad de los estudiantes.

Con respecto a los resultados de la dimensión de la capacidad de respuesta, se concluye que si se mejora la rapidez de atención y la atención de quejas a un nivel de excelente, la probabilidad de que los estudiantes sean apóstoles es del $50.6 \%$ y de que sean leales 42.7 , que el estudiante este en un estado de terrorista es solo del 6.67.

Con respecto a los resultados de la dimensión de empatía del personal, se concluye que si se mejora la amabilidad y cortesía, y escuchar al alumno a un nivel de excelente; la probabilidad de que los estudiantes sean apóstoles es del $50.3 \%$, sean leales 43.4, y el estudiante en estado de terrorista es del 6.36 .

Con respecto a los resultados de la dimensión de seguridad concluye que si el estudiante está conforme con el servicio, recibe información útil, percibe seguridad en el campus, la orientación es calificada como excelente y el desempeño del docente es óptimo, entonces la probabilidad de que los estudiantes sean apóstoles es del $57.3 \%$, sean leales $36.5 \%$, y que el estudiante este en un estado de terrorista es de 6.29.

Con respecto a los resultados de la dimensión de fiabilidad del servicio, se concluye que si se comunica de manera clara donde debe quejarse el alumno y el alumno califica como excelente este factor, la probabilidad de que los estudiantes sean apóstoles es del $50.3 \%$, sean leales 43.4 y que el estudiante este en un estado de terrorista es de 6.29.

Se recomienda la capacitación que debe estar enfocada en tres factores amabilidad, cortesía y saber escuchar al alumno. El hecho de enfocarse en estos tres factores se tendrá una alta probabilidad de que el estudiante sea un apóstol.

Se recomienda con respecto a la dimensión de fiabilidad del servicio, y de acuerdo al modelo Serqual hacer énfasis en comunicar al estudiante de manera clara donde debe quejarse ya que es una de los factores de mayor efecto en la lealtad.

Se recomienda al personal de limpieza cumplir con el plan de mejoras ya que este factor tiene una influencia positiva en la lealtad de los estudiantes. También es necesario mejorar la comodidad del aula y la comodidad de las oficinas ya que estas son también de mayor influencia en la lealtad de los mismos.

Se recomienda a los directivos de la universidad en estudio implementar el sistema de gestión por procesos que permita mejorar la rapidez de atención así como la atención de quejas ya que estos factores son de mayor influencia en la lealtad de los estudiantes.

\section{REFERENCIAS BIBLIOGRÁFICAS}

[1] Berger, J. B., \& Lyon, S. C. (2005). Past to present: a historical look at retention. In A. Seidman (Ed.). In College Student Retention (pp. 1-29). Westport: Praeger Publishers.

[2] Boulding, W., Kalra, A., Staeling, R. \& Zeithaml, A. (1992). A dynamic process model of service quality: from expectations to behavioral intentions. Joumal of Marketing Research, 30, 7-27.

[3] Cheng, P. W. (1997). From covariation to causation: a causal power theory. Psychological Review, 104, 367-405.

[4] Cheng, Q. (1995). The perimeter-area fractal model and its application in geology. Mathematical Geology, 27, 69-82. 
[5] Fuller, B. (1986). Raicing school quality in developing countries, what investments boost learning. World Bank discussion Pappers 2. Washington, D.C.: the world bank.

[6] Hernández Sampieri, Fernández \& Baptista (2010). Metodología de la investigación. México: Editorial McGraw Hill.

[7] Herzog, S. (2005). Medición determinantes del estudiante Devuelve vs. Deserción / Stopout vs. Transferencia: un análisis de la nueva estudiante de primer año del primero al segundo año. Investigación en la Educación, 46 (8), 883-928.

[8] Kotler, P. \& Fox, K. F. A. (1994). Marketing estratégico para instituições educacionais. São Paulo: Atlas.

[10] McLaughlin, G. W., Brozovsky, P. V., \& McLaughlin, J. S. (1998). Changing perspectives on student retention: a role for institutional research. Research in Higher Education, 39(1), 1-17.

[11] Millikan, R. G. (2007). Reply to Bermúdez. Philosophy and phenomenological research, 75, 670-673.

[12] Mortenson, T. G. (2005). Measurements of persistence. In: SEIDMAN, A. (Ed.). In Colle- gestudent retention: formula for student success. Westport: Greenwood Publishing Group.

[13] Newman, J.W. \& Werbal, R. A. (1973). Multivariate analysis of brand loyalty for major household appliances. Journal of Marketing Research, 10, 404-9.

[14] Oliver, R. L. (1999). Whence consumer loyalty? Journal of Marketing (special Issue), 63, 33-44.

[15] Schwartzman, J. S. (2003). Sistema nervoso na Síndrome de Down. In: Síndrome de Down. pp. 44-81. São Paulo: Mackenzie.

[16] Sirdeshmukh, D., Singh, J., \& Sabol, B. (2002). Consumer trust, value, and loyalty in relational exchanges. Journal of Marketing, 66, 15-37.

[17] Parasuraman, A., Zeithaml VA., \& Berry, L. L. (1988). SERVQUAL: a multiple-item scale for measuring consumer perceptions of service quality. Journal of Retailing, 16, 12-40.

[18] Wetzel, J. N., O'Toole, D., \& Peterson, S. (1999). Factors affecting student retention probabilities: a case study. Journal of Economics \& Finance, 23(1), 45-55. 